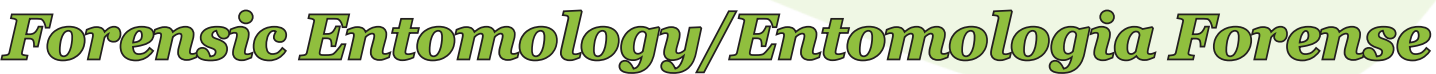

\section{Análise temporal de Calliphoridae (Diptera: Cyclorrhapha) no processo de decomposição em carcaças de suínos (Sus scrofa Linnaeus) em Salvador, Bahia, Brasil}

Fernanda Pamponet ${ }^{1}{ }^{\bowtie}$, Daniele Lopes ${ }^{1}$, Thaís Véras ${ }^{2}$, Patricia Fonseca $^{2}$, Susane Vasconcelos $^{2}$, Mateus Torres $^{2}$, Bianca Grisi² $^{2}$ Favizia Oliveira ${ }^{1}$, Torriceli Thé2 $\&$ Janyra Oliveira-Costa ${ }^{3}$

1. Laboratório de Bionomia, Biogeografia e Sistemática de Insetos (BIOSIS), Departamento de Zoologia, Universidade Federal da Bahia, Salvador, BA. 2. Laboratório Central de Polícia Técnica da Bahia. Departamento de Policia Técnica da Bahia, Salvador, BA. 3. Laboratório de Entomologia Forense, Instituto de Criminalística Carlos Éboli. Rio de Janeiro, RJ.

\section{EntomoBrasilis 12 (2): 63-69 (2019)}

Resumo. Em investigações criminais, a entomologia forense torna-se uma importante ferramenta em uma das suas principais aplicações que são os cálculos de estimativa de intervalo pós-morte (IPM), onde os dípteros da família Calliphoridae constituem uma das principais famílias de interesse forense. O presente trabalho teve como objetivo verificar a ocorrência das espécies desta família durante o processo de decomposição de carcaças suínas exposta durante as quatro estações do ano em um fragmento de Mata Atlântica na cidade do Salvador, Bahia, Brasil. Durante o experimento, os insetos adultos foram capturados em armadilhas específicas, e os imaturos coletados sobre a carcaça e criados para identificação pós-emergência. Além disso, verificamos a correlação entre as espécies que visitam a carcaça com aquelas que se desenvolvem na mesma, relacionando-a as fases da decomposição. Foram coletados 29136 adultos e do total de imaturos coletados apenas 1278 emergiram no laboratório. Os adultos capturados pertenciam às seguintes espécies: Cochliomyia macellaria (Fabricius), Chrysomya megacephala (Fabricius), Chrysomya albiceps (Wiedemann), Chrysomya putoria (Wiedemann), Lucilia eximia (Wiedemann), Mesembrinella bellardiana (Aldrich), Hemilucilia segmentaria (Fabricius) e Chloroprocta idiodea (Robineau-Desvoidy). Os imaturos coletados e criados no laboratório, após a emergência, foram identificados como: $C$. macellaria, C. megacephala, C. albiceps, C. putoria, H. segmentaria e L. eximia. Constatou-se que há diferenças na abundância entre as espécies durante o experimento, sendo a espécie C. albiceps a mais prevalente. Esse trabalho contribuiu com informações essenciais para a implementação deste tipo de perícia na rotina do Laboratório de Entomologia Forense do DPT-BA.

Palavras-chave: Carcaças de suínos; entomologia forense; decomposição cadavérica; fauna cadavérica; Peckia.

\section{Temporal analysis of Calliphoridae (Diptera: Cyclorrhapha) in the process of decomposition in carcasses of swine (Sus scrofa Linnaeus) in Salvador, Bahia, Brazil}

\begin{abstract}
In criminal investigations, Forensic Entomology becomes an important tool for main applications, which are estimates of postmortem interval (IPM), where Diptera of the Calliphoridae family constitute one of the main families of forensic interest. The present work had as objective to verify the occurrence of the species of this family during the decomposition process of swine carcasses exposed during the four seasons of the year in a fragment of Atlantic Forest in the city of Salvador, Bahia, Brazil. During the experiment, adult insects were captured in specific traps, and immature stages collected on the carcass created until the adult emergency for identification. In addition, we verified the correlation between the species that visit the carcass with those that develop in the same, related to the phases of the decomposition. A total of 29136 adults were collected and of the total number of immatures collected, only 1278 emerged in the laboratory. The captured adults belonged to the following species: Cochliomyia macellaria (Fabricius), Chrysomya megacephala (Fabricius), Chrysomya albiceps (Wiedemann), Chrysomya putoria (Wiedemann), Lucilia eximia (Wiedemann), Mesembrinella bellardiana (Aldrich), Hemilucilia segmentaria (Fabricius) and Chloroprocta idiodea (RobineauDesvoidy). The immatures collected and created in the laboratory after the emergency were identified as: C. macellaria, C. megacephala, C. albiceps, C. putoria, $H$. segmentaria and L. eximia. It was verified that there are differences in the abundance between the species during the experiment, being the species C. albiceps the most prevalent. This work contributed with essential information for the implementation of this type of expertise in the routine of the Forensic Entomology Laboratory, DPT-BA.
\end{abstract}

Keywords: Cadaveric decomposition; cadaverous fauna; carcasses of swine; Forensic entomology; Peckia.

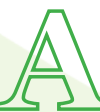

Entomologia Forense constitui uma importante ferramenta em investigações criminais principalmente a médico-legal, relacionada a casos de morte violenta.
Isso ocorre porque a ação dos insetos necrófagos, que utilizam a matéria orgânica em decomposição, como fonte nutricional para estimular a postura ou para desenvolvimento de suas fases
Edited by:

William Costa Rodrigues

\section{Article History:}

Received: 20. iii.2019

Accepted: 25.v.2019
Corresponding author:

Fernanda Pamponet

乃 nandapamponet@gmail.com

D No ORCID record
Funding agencies:

¿ Fundação de Amparo à Pesquisas do Estado da Bahia (FAPESB) ; Laboratório Central da Policia Técnica - DPT/BA; $51^{\circ}$ Centro de Telemática na $6^{a}$ Região do Exercito, Quartel do Imbui. 
imaturas, apresentam um fundamental papel na decomposição de corpos (Moura 2004; Mise et al. 2007; OliveIRA-CoSTA 2011).

A aplicação médico-legal do estudo desses insetos justifica-se no seu pioneirismo no processo de decomposição (a colonização por insetos ocorre logo após a morte), sua presença em todos os estágios de decomposição além da especificidade de algumas espécies a determinadas áreas geográficas e estações do ano (CARvalho et al. 2000; CAMPOBASSO et al 2001; TuRChETto \& VANIN 2004; PUJOL-LUZ et al. 2008).

Dentre as possíveis aplicações médico-legais, os insetos permitem a estimativa do intervalo pós-morte (IPM) com acurácia. Essa estimativa tanto pode ser baseada na sucessão de diferentes espécies durante o processo de decomposição (IPM máximo) quanto no tempo em que o inseto leva para se desenvolver de ovo ou larva até o estágio encontrado no cadáver ou local de crime (IPM mínimo) (WOLFF et al. 2001; ADAMS \& HALL 2003; MiRANDA et al. 2006).

Na sucessão dos insetos, cada espécie tem preferência por determinada etapa da decomposição, pois cada uma delas oferece condições ideais para o desenvolvimento de determinados grupos de insetos (OliveIra-Costa, 2011; PINHEIRo et al. 2012).

$\mathrm{Na}$ entomofauna cadavérica, as ordens Diptera e Coleoptera são os principais grupos de interesse forense (CARVALHO \& LINHARES 2001; TURCHETTO \& VANIN 2004). De acordo com GrEenberg (1991), CATTS \& GofF (1992), CARVAlHo et al. (2000) e CAMPOBASSO et al. (2001) na ordem Diptera, as principais famílias são Calliphoridae, Sarcophagidae e Muscidae. Os califorídeos, foco desse trabalho, são considerados como o grupo de grande importância forense, visto que figura entre os primeiros insetos a localizarem a carcaça, desenvolvem-se nela e são encontrados durante todo o processo de decomposição (CAMPOBASSO et al. 2001; CARVALHO \& LINHARES 2001; AMENDT et al. 2004). Consequentemente, para aplicação da técnica entomológica nos exames periciais é primordial que seja conhecida à fauna de califorídeos associados aos corpos em processo de decomposição.

$\mathrm{Na}$ Bahia, as pesquisas em entomologia forense iniciaram com os registros feitos pelo Professor e Médico Legista Oscar Freire no ano 1914, ocorrendo uma pausa de quase 90 anos, e a partir dos anos 2000, com a fundação do Laboratório de Entomologia Forense no Departamento de Polícia Técnica DPT/BA e parceria com a Universidade Federal da Bahia (UFBA), foram retomadas pesquisas na área o que caracteriza a importância na atualização dos dados entomológicos dessa região.

Desta forma, o presente trabalho tem por objetivo verificar a ocorrência de dípteros da família Calliphoridae ao longo do processo de decomposição de carcaças de suínos (Sus scrofa Linnaeus) exposta durante as estações do ano em um fragmento de Mata Atlântica, associando a ocorrência destes aos estágios de decomposição, além de correlacionar as espécies que visitam a carcaça com àquelas que se desenvolveram na mesma.

\section{MATERIAL E MÉTODOS}

O experimento foi realizado em um fragmento de Mata Atlântica no $51^{\circ}$ Centro de Telemática, localizado no Quartel da Polícia do Exército situado na cidade do Salvador, Bahia ( $12^{\circ} 58^{\prime} 27,54^{\prime \prime}$; $38^{\circ} 26^{\prime} 47,68^{\prime \prime}$ W).

O experimento ocorreu no ano de 2009, durante 31 dias nas quatro estações do ano: Verão (07 de janeiro a 07 de fevereiro), Outono (13 de abril a 14 de maio), Inverno (26 de junho a 26 de julho) e Primavera (14 de outubro a 14 de novembro).

Foram utilizados como atrativos para os insetos quatro suínos adultos (S. scrofa) de aproximadamente $18 \mathrm{Kg}$. A eutanásia dos modelos ocorreu por disparo de arma de fogo, no local do experimento como recomendado pelo Comitê de Ética em experimentação animal da Universidade Estadual de Feira de Santana - UEFS.

Para classificação das fases de decomposição foram adotadas cinco etapas como proposto por BoRNEMISSZA (1957).

As carcaças foram colocadas sobre o solo e cobertos por uma armadilha tipo Shannon modificada. No topo da armadilha foi deixada uma abertura para acoplamento de um "recipiente coletor", para captura dos insetos que, visitavam a carcaça. (Oliveira-Costa 2007, 2011).

Os imaturos foram coletados sobre a carcaça ou no solo e transportados em recipiente com gelo químico. Todo o material coletado foi encaminhado ao Laboratório de Entomologia Forense no Laboratório Central de Polícia Técnica, DPT-BA.

A identificação dos insetos da família Calliphoridae foi feita até espécie, utilizando chaves taxonômicas: RIBEIRo \& CARVALHO (2000); Mello (2003) e Carvalho \& Mello-Patiu (2008).

\section{RESULTADOS E DISCUSSÃO}

Nas quatro estações do ano, a duração dos estágios de decomposição foi semelhante (Figura 1). A primeira fase de decomposição, (fase fresca) teve a mesma duração em todos as estações do ano (1 dia), onde a carcaça encontrou-se fresca externamente, mas já se inicia a decomposição interna resultante da ação de microrganismos presentes no corpo antes da morte (BORNEMISSZA 1957; BURKEPILE et al. 2006). Os primeiros insetos a chegarem à carcaça levam pouco tempo e são atraídas pelo sangue e outras secreções orgânicas de orifícios naturais ou artificiais.

A segunda fase (fase gasosa) apresentou duração de 1 dia no verão e no outono e de dois dias no inverno e na primavera. Essa fase foi caracterizada pelo inchamento da carcaça causado, principalmente, pelo acúmulo de gases oriundos da decomposição interna por bactérias anaeróbias (BURKEPILE et al. 2006). Nessa etapa pôde ser observada a preferência dos dípteros em ovipositar, principalmente, nos orifícios naturais (boca, nariz, ouvido e ânus) e no ferimento, corroborando com os resultados observados nos trabalhos de HASKell et al. (1997).

A terceira etapa (fase de deterioração inicial) apresentou duração de dois dias no outono e um dia nas demais estações. Observouse nessa fase uma decomposição ativa da carcaça com a ruptura do tegumento abdominal, as partes expostas apresentando-se escurecidas e com aspecto cremoso (BoRNEMIsszA 1957). Nessa fase notou-se odor muito forte e uma intensa atividade das larvas necrófagas.

Na quarta etapa (fase de deterioração avançada) o processo de decomposição teve duração de 2 dias no verão, 4 dias no outono e no inverno e 6 dias na primavera. Nesta fase, a carcaça apresentou-se em decomposição avançada, secando por fora e ausência de odor. Observou-se ainda que as larvas de dípteros estavam menos ativas, começavam a dispersar-se em busca do solo, o que caracteriza o início da pupação. De acordo com GREENBERG (1990), a dispersão larval é uma característica observada no momento em que as larvas estão procurando um local para pupação.

$\mathrm{Na}$ última etapa (fase restos), em todos os períodos estudados, considerando o tempo de acompanhamento do experimento (31 dias), o processo de decomposição apresentou valores entre 21 e 26 dias. Observou-se a carcaça estava seca, com presença de ossos expostos e fragmentos do couro da região lombar. Nesta fase, observou-se a presença de coleópteros e pupas de dípteros. Concordando com GoFF (1991) e CRUZ \& VASCONCELOS (2006) que também encontraram, em seus trabalhos, maior ocorrência 




Figura 1. Duração (dias) das fases de decomposição das carcaças de suínos durante as quatro estações estudadas na cidade do Salvador, Bahia, Brasil.

de coleópteros em estágios mais tardios da decomposição. Nesse contexto, KulShresthA \& SATPATHY (2001) relataram que em esqueletos secos de humanos que são encontrados, os Coleoptera (besouros) compreendem a principal evidência entomológica na determinação do IPM, baseada principalmente no padrão de sucessão.

Durante o processo de decomposição foram capturados 29.136 califorídeos adultos (Tabela 1), sendo 66\% fêmeas (19.099 espécimes) e 34\% machos (10.037 espécimes) (Figura 2). A maior ocorrência de fêmeas foi observada nos três primeiros estágios de decomposição em todas as estações do ano, o que reforça a ideia de que essas espécies são atraídas para a carcaça visando a oviposição (MENDES \& LinHARES 1993; MARCHENKO 2001). Essas três primeiras fases estão relacionadas também à quantidade de califorídeos que foram atraídos para a carcaça. Autores como SMITH (1986) e AMENDT et al. (2004) verificaram que esta família predomina desde os primeiros momentos após a morte até quando os tecidos moles são deteriorados.

Verificamos neste trabalho que a emergência da primeira geração de califorídeos que completou o seu ciclo biológico a partir dos ovos colocados sobre a carcaça nos estágios iniciais ocorreu coincidindo com a fase restos (Figura 2).

As variações meteorológicas podem ter influenciado a estrutura da fauna decompositora de forma qualitativa e quantitativa. A temperatura média e a umidade relativa do ar, durante as estações estudadas não demonstraram grande variação, merecendo destaque para o Verão que, por apresentar maior temperatura média, observou-se um maior número de califorídeos coletados (11.566 espécimes). Esse fato também foi observado por SousA \& LiNHARES (1997) que afirmaram que, no Verão, período em que a temperatura e umidade relativa são elevadas, observa-se uma diversidade e abundância maior de espécies de insetos, resultando em intensa atividade metabólica, permitindo um acelerado processo de decomposição.

Dentre as espécies coletadas, Chrysomya albiceps (Wiedemann) apresentou maior abundância (22.364 indivíduos) em todos os estágios de decomposição, exceto nos estágios IV e V na Primavera (Tabela 1), assim como foi o mais abundante dentre os imaturos coletados e posteriormente criados em laboratório $(84,3 \%)$ (Tabela 2). Segundo Rodrigues-GuimarãEs et al. (2004) a grande abundância dessa espécies deve-se ao fato de apresentarem alta capacidade de dispersão, alto crescimento populacional, alta capacidade reprodutiva, curto ciclo de vida, aliado ao fato da mesma ser predadora de larvas, o que resulta estar entre as primeiras espécies que chegam à carcaça.

O predomínio de emergências de C. albiceps, segundo HoRENSTEIN et al. (2007) pode ser explicado pelo fato de que a presença desses imaturos pode influenciar a oviposição de outros califorídeos, os quais podem escolher locais com baixo risco de predação. Além disso, alguns autores consideraram a predação larval como uma interação capaz de influenciar a composição da fauna necrófaga (BAz et al. 2007), o que pode interferir na diversidade de insetos encontrados, conforme observado na presente pesquisa.

Chrysomya megacephala (Fabricius), segunda espécie mais abundante (2.714 espécimes) teve a sua maior incidência observada na fase III no Verão. Segundo OliveIRA-Costa (2007), C. megacephala, junto com C. albiceps, aparece em maior número quando a carcaça começa a exalar os odores da fermentação, contudo nesse trabalho seu pico ocorreu no estágio anterior.

A terceira espécie mais abundante foi Chloroprocta idioidea (Robineau-. Desvoidy) (1.554 espécimes) ocorrendo no Inverno e Primavera, sendo observada sua maior incidência na fase $\mathrm{V}$. De acordo com PARALUPPI (1996), a ocorrência de C. idioidea no Inverno e Primavera pode ser explicada pela média de umidade relativa do ar (71\% e 61\%), pois, em seus trabalhos, encontrou grandes picos desta espécie coincidindo com as épocas de maiores pluviosidades.

Chrysomya putoria (Wiedemann) foi a quarta espécie mais frequente (795 espécimes), ocorrendo em todas as fases nas quatro estações, exceto na fase I na Primavera. Suas maiores ocorrências foram observadas nas fases II e III no Outono e fase II no Inverno, contudo, obteve pouco sucesso de emergência.

Foram registrados 494 espécimes de Hemilucilia segmentaria (Fabricius) (Tabela 1). Essa espécie ocorreu, principalmente, nas duas primeiras fases em todas as estações do ano. A espécie $H$. segmentaria apresentou sucesso reprodutivo com o segundo percentual em emergências (11,4\%) (Tabela 2) corroborando com o trabalho de RosA et al. (2009) que constatou a utilização da carcaça por essa espécie como substrato de criação de larvas.

Lucilia eximia (Wiedemann) foi a sexta espécie mais abundante (477 espécimes) apresentando pioneirismo no abandono da carcaça similar ao verificado por GIÃo \& GoDOY (2006) e HoRENSTEIN et al. (2007), os quais observaram um 




Figura 2. Califorídeos adultos capturados com a armadilha conforme modelo proposto por OliveIRA-Costa (2011), durante as quatro estações do ano estudas na cidade do Salvador, Bahia, Brasil.

comportamento pioneiro no processo de sucessão entomológica, deixando a carcaça mais cedo que as outras espécies, evitando, assim, a predação de larvas de terceiro instar por C. albiceps. A ocorrência dessa espécie no início da fase de restos (Tabela 1) sugere que os adultos aqui coletados, são resultantes de oviposições nas etapas inicias de decomposição, visto que, quando coletados estavam no terceiro instar larval. Das espécies criadas em laboratório, L. eximia obteve sucesso de emergência correspondente às fases I, II, III e V (Tabela 2).

A sétima espécie mais abundante foi Mesembrinella bellardiana (Aldrich) (461 espécimes) ocorrendo em todas as fases nas diferentes estações do ano. Não foi possível observar estágios imaturos desta espécie na carcaça (Tabela 2), pois segundo Mello (2003), as fêmeas desse gênero não ovipositam, elas liberam uma larva de terceiro instar que passa ao estágio de pupa imediatamente.

Cochliomyia macellaria (Fabricius) foi a espécie menos frequente (277 espécimes coletados), e muitos autores atribuem sua diminuta ocorrência ao impacto causado pela introdução de espécies de Chrysomya (GUIMARÃes et. al. 1978 e HoRENSTEIN et al. 2007). No presente trabalho, apenas um exemplar dessa espécie emergiu no laboratório (Tabela 2).

Os califorídeos imaturos totalizaram 1324 indivíduos coletados no Verão, 552 indivíduos no Outono; 648 indivíduos coletados no Inverno e 361 na Primavera, dos quais apenas emergiram em laboratório 537, 273, 309 e 159 respectivamente (Tabela 2). O insucesso nas emergências no Verão, Outono, Inverno e Primavera foram, aproximadamente, de $59 \%, 51 \%$, 52\% e $56 \%$, respectivamente, eforam atribuídos a fatores como mortalidade de larvas, pupas fechadas com adultos mortos, pupas sem completar o desenvolvimento (devido ao parasitoidismo registrado), além de adultos recém-emergidos com morte precoce (Figura 3).

O estudo de espécimes da Família Calliphoridae associados à decomposição de carcaças é importante quando se pretende acumular informações sobre a biologia e comportamento desses insetos, visando sua utilização em investigações periciais e estimativas de intervalo pós-morte, conforme já vem sendo feito na rotina do Laboratório de Entomologia Forense do DPT-BA

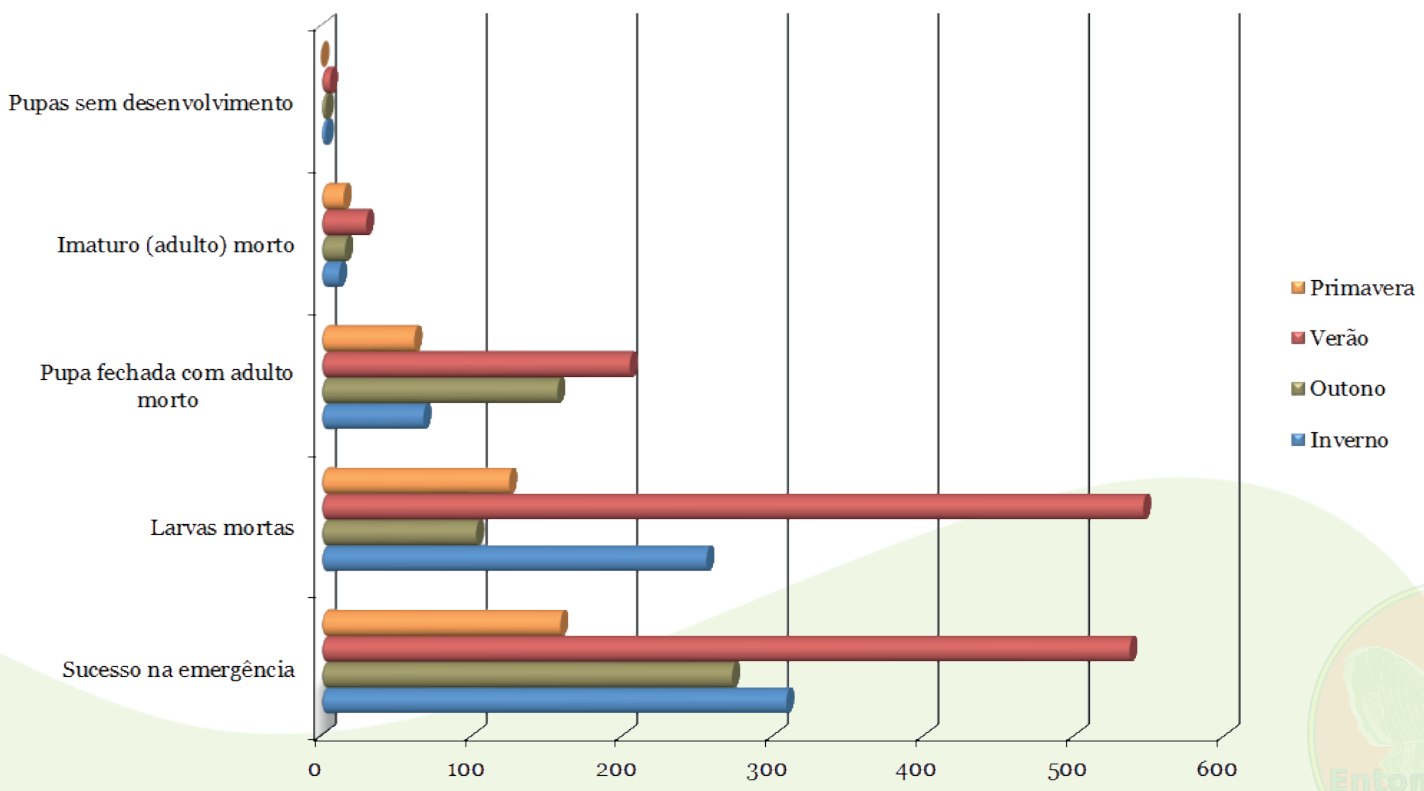

Figura 3. Fatores relacionados à criação dos imaturos de califorídeos criados em laboratório durante o experimento realizado na cidade do Salvador, Bahia, Brasil. 

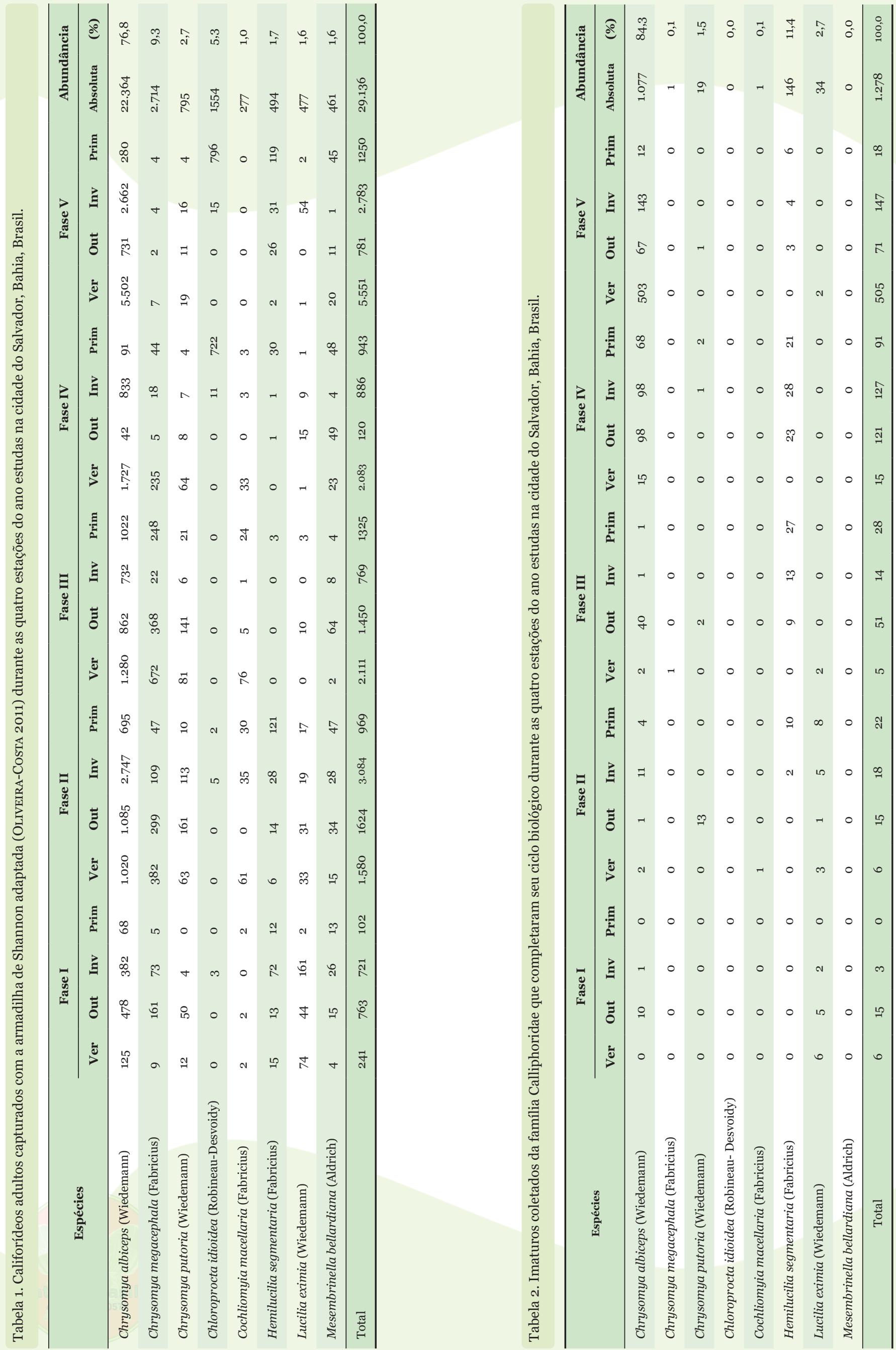
e Laboratório BIOSIS-UFBA, em Salvador (Bahia), graças aos resultados experimentais preliminares embasadores já obtidos, foi possível o início da formação de um banco de dados necessário às perícias e a aplicabilidade prática destas informações, sobretudo em investigações criminais.

\section{AGRADECIMENTOS}

À Fundação de Amparo à Pesquisas do Estado da Bahia (FAPESB) pelos investimentos concedidos ao projeto, ao Laboratório Central da Policia Técnica - DPT/BA pelo apoio dado para execução das pesquisas e ao $51^{\circ}$ Centro de Telemática na $6^{\mathrm{a}}$ Região do Exercito, Quartel do Imbui, pela permissão concedida para realização deste trabalho nas suas dependências.

\section{REFERÊNCIAS}

Adams, ZJO \& MJR Hall, 2003. Methods used for the killing and preservation of blowfly larvae, and their effect on postmortem larval length. Forensic Science International, 138: 5061. DOI: https://doi.org/10.1016/j.forsciint.2003.08.010.

Amendt, J, R Krettek \& R Zehner, 2004. Forensic Entomology. Naturwissenschaften, 91: 51-65. DOI: https://doi.org/10.1007/s00114-003-0493-5.

Baz, A, B Cifrián, LM Díaz-Aranda \& D Martínveja, 2007. The distribution of adult blow-flies (Díptera: Calliphoridae) along na altitudinal gradient in Central Spain. Annales de la Société entomologique de France (N.S.), 43: 289-296. DOI: https://doi.org/10.1080/00379271.2007.10697524.

Bornemissza, GF, 1957. Analysis of arthropod succession in carrion and the effect of its decomposition on the soil fauna. Australian Journal of Zoology, 5: 1-12. DOI: https://doi.org/10.1071/z09570001.

Burkepile, DE, JD Parker, CB Woodson, HJ Mills, J Kubanek, PA Sobecky \& ME Hay, 2006. Chemically mediated competition between microbes and animals: microbes as consumers in food webs. Ecology, 87: 2821-2831. DOI: https://doi. org/10.1890/0012-9658(2006)87[2821:cmcbma]2.0.co;2.

Campobasso, CPG, D Vella \& F Introna, 2001. Factors affecting decomposition and Diptera colonization. Forensic Science International, 120: 18-27. DOI: https://doi.org/10.1016/ S0379-0738(01)00411-X.

Carvalho, CJB \& CA Mello-Patiu, 2008. Key to the adults of the most common forensic species of Diptera in South America. Revista Brasileira de Entomologia, 52: 390-406. DOI: https://doi.org/10.1590/s0085-56262008000300012.

Carvalho, LML \& AX Linhares, 2001. Seasonality of Insect Succession and Pig Carcass Decomposition in a Natural Forest Area in Southeastern Brazil. Journal of Forensic Sciences, 46: 604-608. DOI: https://doi.org/10.1520/jfs15011j.

Carvalho, LML, PJ Thyssen, AX Linhares \& FAB Palhares, 2000. A Checklist of Arthropods associated with Pig Carrion and Human Corpses in Southeastern Brazil. Memórias do Instituto Oswaldo Cruz, 95: 135-138. DOI: https://doi.org/10.1590/ s0074-02762000000100023.

Catts, EP \& ML Goff, 1992. Forensic entomology in criminal investigations. Annual Review of Entomology, 27: 253-272. DOI: https://doi.org/10.1146/annurev.ento.37.1.253.

Cruz, TM \& S.D. Vasconcelos, 2006. Entomofauna de solo associada à decomposição de carcaça de suíno em um fragmento de Mata Atlântica de Pernambuco, Brasil. Biociências, 14: 193-201.

Gião, JZ \& WAC Godoy, 2006. Seasonal Population Dynamics in Lucilia eximia (Wiedemann) (Diptera: Calliphoridae). Neotropical Entomology 35(6):753-756 (2006). DOI: https://doi.org/10.1590/s1519-566x2006000600005.

Goff, ML, 1991.Comparison of Insect Species Associated with Decomposing Remains Recovered Inside Dwellings and Outdoors on the Island of Oahu, Hawaii. Journal of Forensic Sciences, 36: 748-753, DOI: https://doi.org/10.1520/ JFS13085J.
Greenberg, B, 1990. Behavior of postfeeding larvae of some Calliphoridae and a muscid (Diptera). Annals of the Entomological Society of America. 83: 1210-1214. DOI: https://doi.org/10.1093/aesa/83.6.1210.

Greenberg, B, 1991. Flies as forensic indicators. Journal of Medical Entomology, 28: 565-577. DOI: https://doi.org/10.1093/ jmedent/28.5.565.

Guimarães, JH, AP Prado \& A.K Linhares, 1978. Three newly introduced blowfly species in Southern Brazil (Diptera: Calliphoridae). Revista Brasileira de Entomologia, 22: 53-60

Haskell, NH, RD Hall, VJ Cervenka \& MA Clark, 1997. On the body: insects' life stage presence and their postmortem artifacts, pp. 415-448 In: Haglund WD \& MH Sorg (eds.), Forensic Taphonomy: he Postmortem Fate of Human Remains. CRC Press, Inc., Boca Raton.

Horenstein, MB, AX Linhares, B Rosso \& MD Garcìa, 2007. Species composition and seasonal sucession of saprophagous calliphorids in a rural area of Córdoba, Argentina. Biological Research, 40: 163-171. DOI: https://doi.org/10.4067/s071697602007000200007 .

Kulshrestha, P \& DK Satpathy, 2001. Use of beetles in forensic entomology. Forensic Science International 120: 15-17. DOI: https://doi.org/10.1016/s0379-0738(01)00410-8.

Marchenko, MI, 2001. Medicolegal relevance of cadaver entomofauna for the determination of the time of death. Forensic Science International, 120: 89-109. DOI: https://doi.org/10.1016/s0379-0738(01)00416-9.

Mello, RP, 2003. Chave para identificação das formas adultas das espécies da família Calliphoridae (Diptera, Brachycera, Cyclorrhapha) encontradas no Brasil. Entomologiay Vectores, 10: $255-268$.

Mendes, J \& AX Linhares, 1993. Atratividade por iscas e estágios de desenvolvimento ovariano em várias espécies sinantrópicas de Calliphoridae (Diptera). Revista Brasileira de Entomologia, 37: 157-166.

Miranda, G, G Jacques, MP Almeida \& MSB Silva. 2006. Coleta de Amostras de Insetos para Fins Forenses. Brasília, DF: Departamento de Policia Federal. Instituto Nacional de Criminalística. 11 p.

Mise, KM, LM Almeida \& MO Moura, 2007. Levantamento da fauna de Coleoptera que habita a carcaça de Sus scrofa L., em Curitiba, Paraná. Revista Brasileira de Entomologia, 51: 358-368. DOI: https://doi.org/10.1590/s008556262007000300014 .

Moura, MO, 2004. Variação espacial como mecanismo promotor da coexistência em comunidades de insetos necrófagos. Revista Brasileira de Zoologia 21: 409-419. DOI: https://doi.org/10.1590/s0101-81752004000300001.

Oliveira-Costa, J, 2007. Entomologia Forense: quando os insetos são vestígios. Campinas, SP: Millennium Editora, 420 p.

Oliveira-Costa, J, 2011. Entomologia Forense: quando os insetos são vestígios. 3 ed. São Paulo: Millennium Editora, 486 p.

Paraluppi, ND, 1996. Calliphoridae (Diptera) da Bacia do Alto Rio Urucu, Amazônia Central, Brasil. Revista Brasileira de Zoologia, 13: 553-559. DOI: https://doi.org/10.159o/s010181751996000300003.

Pinheiro, DS, AAS Reis, RSA Jesuíno \& HMV Silva, 2012. Variáveis na estimativa do intervalo pós-morte por métodos de entomologia forense. Enciclopédia Biosfera, Centro Científico Conhecer - Goiânia, 8:1443.

Pujol-Luz, JR, LC Arantes \& R Constantino, 2008. Cem anos da entomologia Forense no Brasil (1908-2008). Revista Brasileira de Entomologia, 52: 485-492. DOI: https://doi.org/10.1590/s0085-56262008000400001.

Ribeiro, PB \& CJB Carvalho, 2000. Chave de identificação das espécies de Calliphoridae (Diptera) do sul do Brasil. Revista Brasileira de Parasitologia Veterinária, 9: 169-173.

Rodrigues-Guimarães, R, GEM Borja, EA Pile, RR Guimarães \& FR Sampaio, 2004. Constande coefficient of blowflies (Diptera: Calliphoridae) in Nova Iguaçu, Rio de Janeiro, Brazil. Boletin de la S.E.A., 35: 251-254. 
Rosa, TA, MLY Babata, CM Souza, D Sousa. CA Mello-Patiu \& J Mendes, 2009. Dípteros de interesse forense em dois perfis de vegetação de cerrado em Uberlândia, MG. Neotropical Entomology, 38: 859-866. DOI: https://doi.org/10.1590/ S1519-566x2009000600022.

Smith, KGV, 1986. A manual of Forensic Entomology, Cornell Univ. Press Ithaca, NY, 205 p.

Souza, AM \& AX Linhares, 1997. Díptera and Coleóptera of potential forensic importance in southeastern Brazil: relative abundance and seasonality. Medical and Veterinary Entomology 11:8-12. DOI: https://doi.org/10.1111/j.13652915.1997.tboo284.x.
Turchetto, M \& S Vanin, 2004. Forensic entomology and climatic change. Forensic Science International, 146: 207-209, 2004. DOI: https://doi.org/10.1016/j.forsciint.2004.09.064.

Wolff, M, A Uribe, A. Ortiz \& P Duque, 2001. A preliminary study of forensic entomology in Medellín, Colombia. Forensic Science International, 120: 53-59. DOI: https://doi.org/10.1016/ s0379-0738(01)00422-4.

$* * * * * * * * * *$

\section{Suggestion citation:}

Pamponet, F, D Lopes, T Véras, P Fonseca, S Vasconcelos, M Torres, B Grisi, F Oliveira, T Thé \& J Oliveira-Costa, 2019. Análise temporal de Calliphoridae (Diptera: Cyclorrhapha) no processo de decomposição em carcaças de suínos (Sus scrofa Linnaeus) em Salvador, Bahia, Brasil. EntomoBrasilis, 12 (2): 63-69.

Available on: doi:10.12741/ebrasilis.v12i2.846

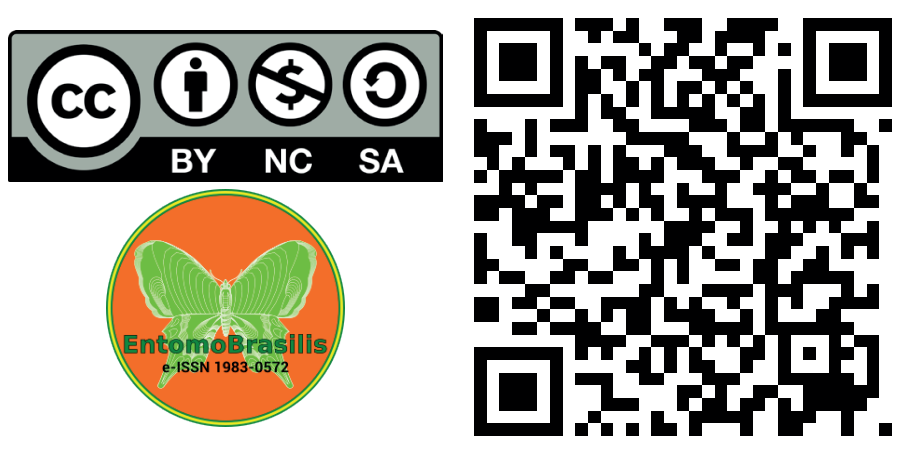

